2-14 Wakamiyacho, Nakamura-ku, Nagoya, Aichi 453-0023,

Japan. E-mail: crimson@dk2.so-net.ne.jp

Statement of Interest: None declared.

\section{ACKNOWLEDGEMENTS}

The authors thank K. Oshima for data analyses.

\section{REFERENCES}

1 Teramoto S, Matsuse T, Fukuchi Y, et al. Simple two-step swallowing provocation test for elderly patients with aspiration pneumonia. Lancet 1999; 353: 1243.
2 Oguchi K, Saitoh E, Baba M, et al. The repetitive saliva swallowing test (RSST) as a screening test of functional dysphagia. (2) Validity of RSST. Jpn J Rehabil Med 2000; 37: 383-388.

3 Kobayashi S, Kubo H, Yanai M. Impairment of the swallowing reflex in exacerbations of COPD. Thorax 2007; 62: 1017.

4 Mokhlesi B, Logemann JA, Rademaker AW, et al. Oropharyngeal deglutition in stable COPD. Chest 2002; 121: 361-369.

5 www.goldcopd.com GOLD Global Strategy for the Diagnosis, Management, and Prevention of Chronic Obstructive Pulmonary Disease, 2008. Date last updated: January 26, 2009. Date last accessed: January 26, 2009.

\title{
Histological findings of the computed tomography halo in pulmonary sarcoidosis
}

In 1974, SAHN et al. [1] described a patient with sarcoidosis who presented with a fine acinar rosette pattern of infiltrate on chest radiograph and alveolar filling with mononuclear cells in the biopsied specimen. In 1978, SHIGEMATSU et al. [2] reported the presence of noncaseating epithelioid granulomas in the alveolar spaces in patients with radiographic findings similar to the previous report. They considered these lesions to represent the early stage of sarcoidosis. Although there seems to be no definite relationship between the stage of sarcoidosis and the anatomical extent of the granulomas, sarcoid granulomas in the alveoli are rarely found. Nowadays, in the era of high-resolution computed tomography (HRCT), ground-glass attenuations are sometimes found in sarcoidosis that could fit

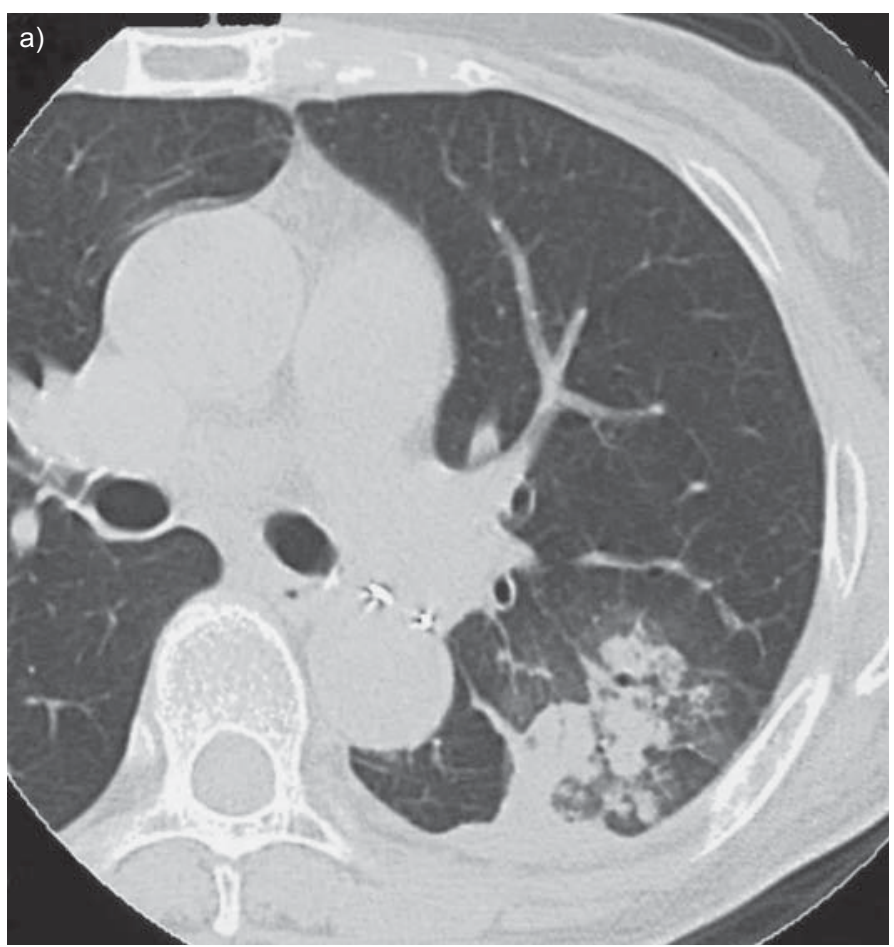

the "acinar pattern" proposed by SAHN et al. [1] and SHIGEMATSU et al. [2].

In 2004, MARTEN et al. [3] described the presence of groundglass attenuation around solid nodules, the halo sign, on computed tomography $(\mathrm{CT})$ of a patient with sarcoidosis.

Herein, we report a case of sarcoidosis presenting a CT halo sign that was surgically biopsied. The pathological/radiological correlation of sarcoidosis with the halo sign is discussed.

A 69-yr-old female noticed general fatigue. She had a past history of left lower lobectomy due to adenocarcinoma of the lung 8 yrs prior to admission. She was admitted to our hospital

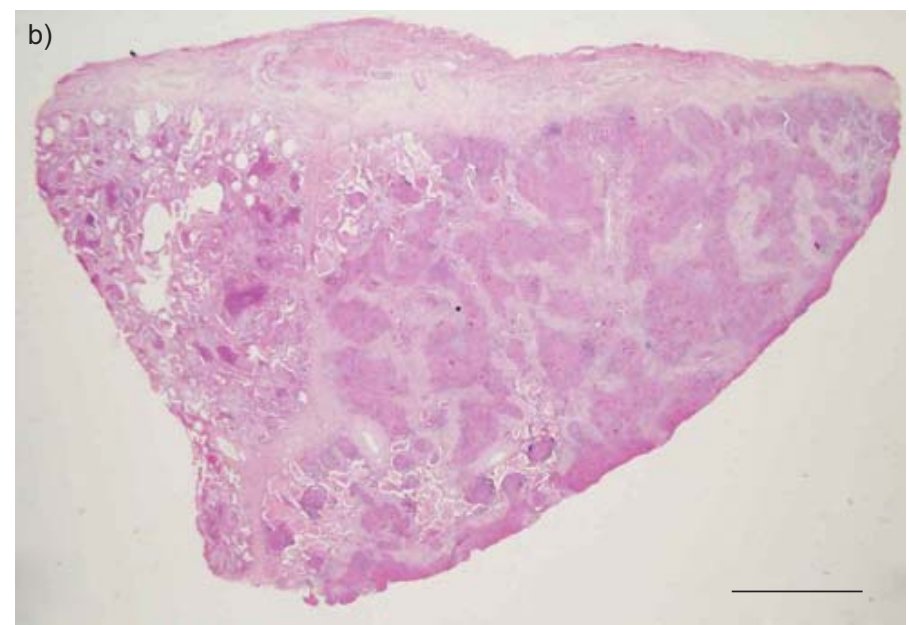

FIGURE 1. a) High-resolution computed tomography (CT) demonstrating multiple nodules and ground-glass opacities surrounding the nodules (i.e. halo sign). b) The biopsied sample on low-power microscopy (haematoxylin and eosin stain). The right two-thirds of the specimen represent the solid area corresponding to an area of the multiple nodules visible on the CT. The left third of the specimen represents the area of the halo sign visible on the CT. Scale bar $=2.0 \mathrm{~mm}$. 
(Fukuoka University School of Medicine, Fukuoka, Japan) and a chest CT showed a nodule attached to the pleura in the left lung. Transbronchial lung biopsy (TBLB) was performed, which revealed the presence of non-necrotising epithelioid granulomas. Holter monitoring electrocardiogram (FT3000 Holter System; Spacelabs Inc., Redwood, WA, USA) for $24 \mathrm{~h}$ found sporadic supraventricular and ventricular arrhythmias, and the skin test against purified protein derivative showed no induration. Sarcoidosis was suspected but serum angiotensin converting enzyme was within normal limits $\left(9.2 \mathrm{U} \cdot \mathrm{mL}^{-1}\right)$. The patient was then discharged from our hospital and a follow-up observation was started in our outpatient clinic. A chest CT scan 6 months after discharge revealed that the nodule had expanded to multiple nodules with surrounding ground-glass opacities in the left lung (fig. 1a). She was readmitted to our hospital and open lung biopsy was performed to obtain a definitive diagnosis.
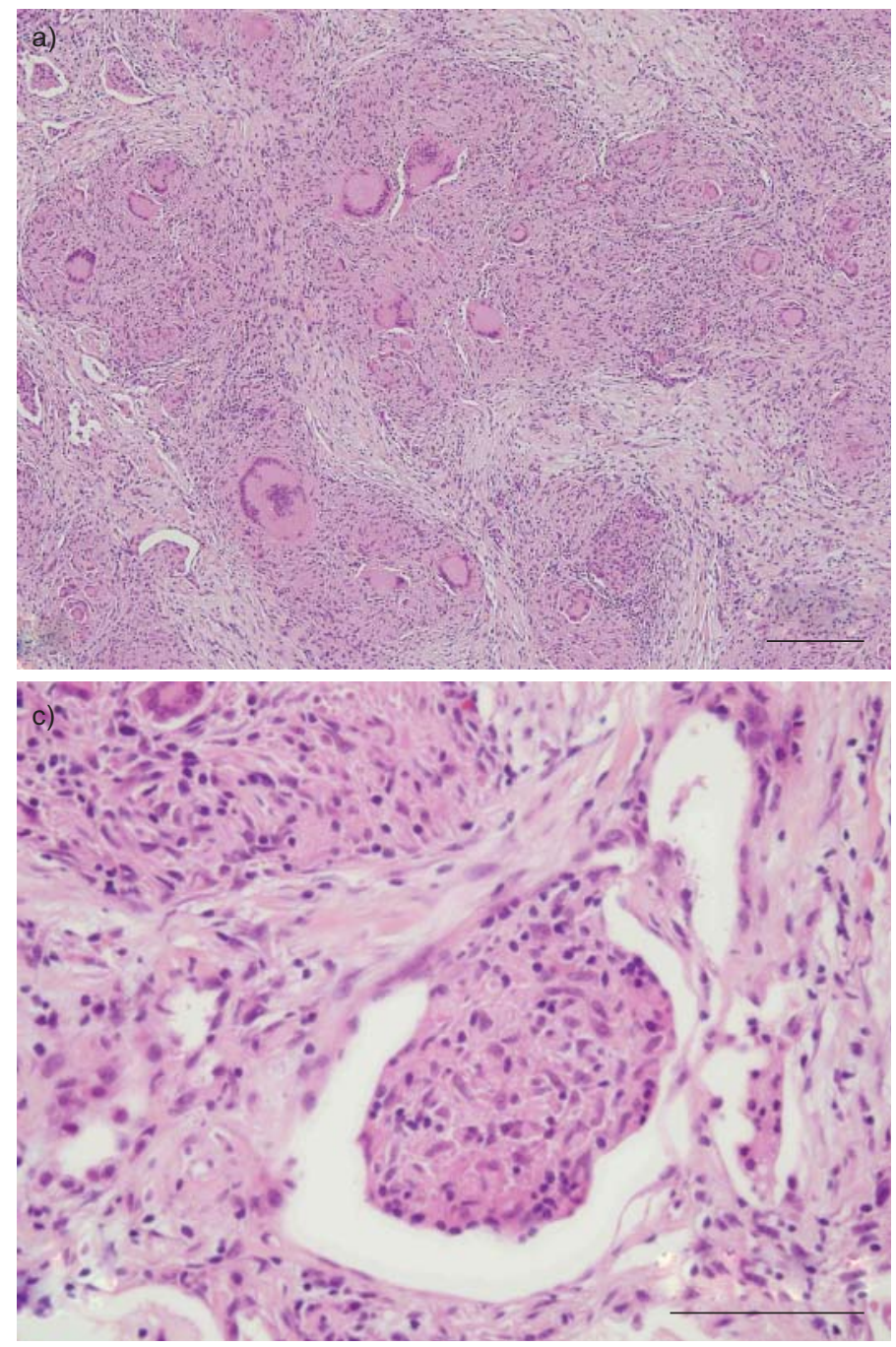

The biopsy specimen (fig. 1b), which was partially resected from the lesion, was composed of two parts. One part consisted of solid areas containing epithelioid cell granulomas intermingled with Langhans' giant cells without caseous necrosis, which are features compatible with sarcoidosis (fig. 2a). The other contained epithelioid granulomas and aggregates of mononuclear cells in the peripheral airways and alveolar spaces with oedematously thickened alveolar septa (fig. 2b). Immunohistochemistry using a CD-68 monoclonal antibody revealed that CD-68 was diffusely expressed in the closely packed mononuclear cells and epithelioid cells that formed granulomas in the alveolar spaces. In addition, some granulomas appeared to be polypoid and were attached to the wall of the peripheral airways (fig. 2c). Immunohistochemistry also revealed that the surface of the intra-alveolar granulomas was partly covered with cytokeratin-7 positive cells (fig. $2 \mathrm{~d}$ ). Neither fungi nor acid-fast bacilli were identified by Grocott
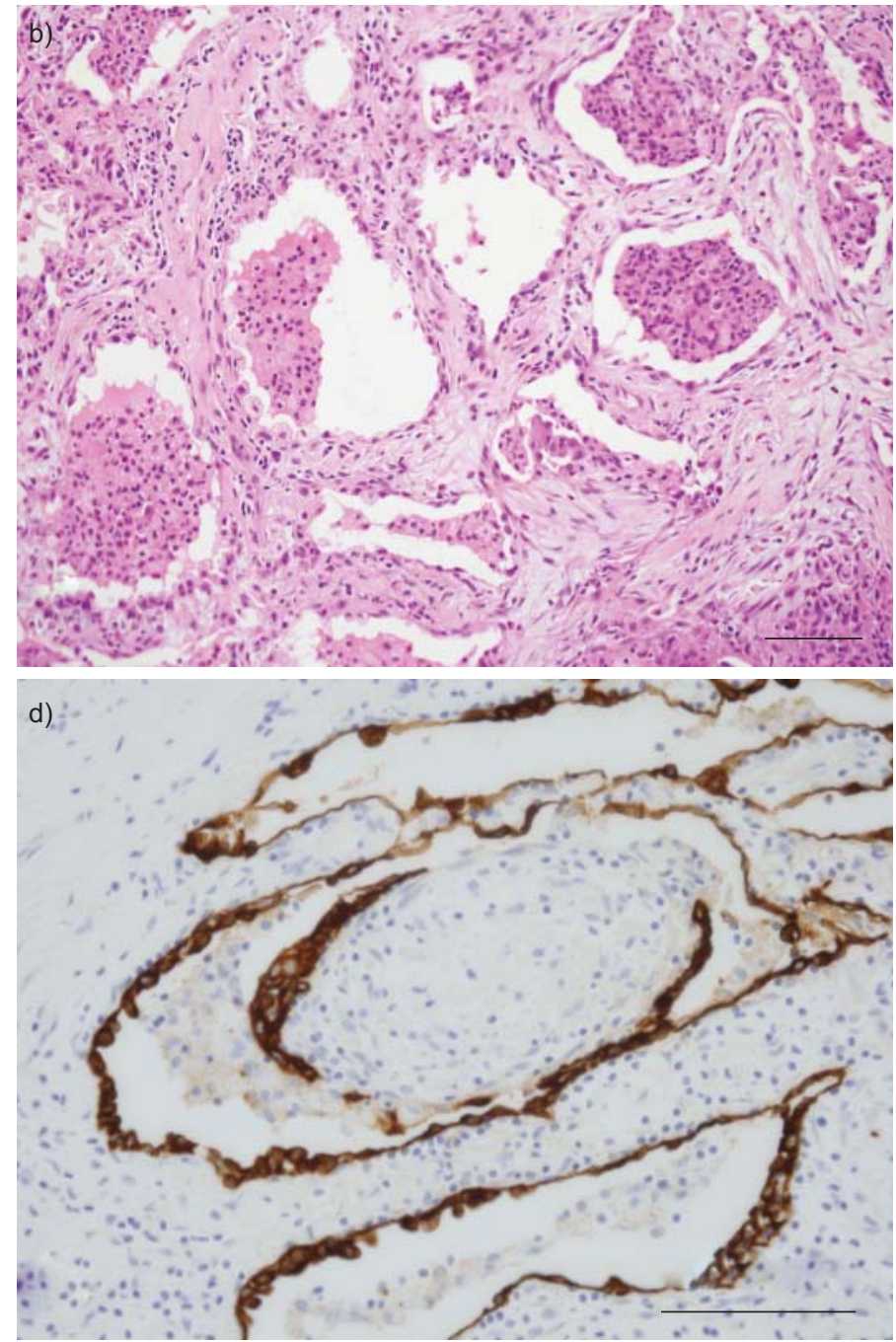

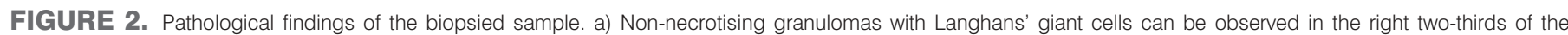

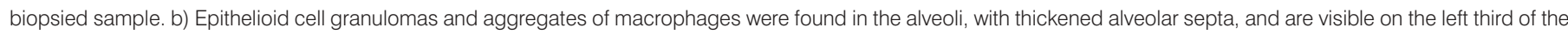

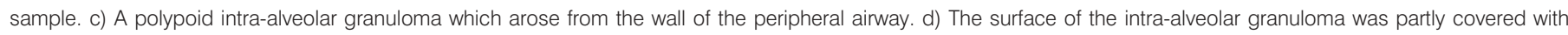
cytokeratin-7 positive cells. a-c) Haematoxylin and eosin stain. a) Scale bar $=200 \mu \mathrm{m}, \mathrm{b}-\mathrm{d}$ ) scale bars $=100 \mu \mathrm{m}$. 
and Ziehl-Neelsen stains, respectively. At that time, the lesions were diagnosed as sarcoidosis.

The patient had adenocarcinoma of the right lower lobe, which was resected 18 months after the biopsy. At that time, the remaining nodular lesions of the left lung had almost disappeared without any treatment. At present, 4 yrs after the biopsy, she is still being followed in our hospital and has had no recurrence.

Alveolitis is histologically defined by a predominantly interstitial accumulation of mononuclear cells (e.g. lymphocytes and macrophages) and is sometimes found in biopsy specimens of sarcoidosis [4]. Together with cellular infiltration in the alveolar spaces, alveolitis was radiographically termed an "acinar pattern". Nowadays, alveolitis is expressed as "ground-glass opacity" on HRCT. Ground-glass opacities have been repeatedly investigated in pulmonary sarcoidosis, which included the examination of the pathological relationship between the CT pattern and histology findings.

In 2004, MARTEN et al. [3] first reported the CT halo sign in pulmonary sarcoidosis; however, histological explanations for the corresponding lesions were not included in that report. In the present study, we reported a patient with sarcoidosis presenting with the CT halo sign and provided details of histology, by showing the transitional zone between the halo sign area and the solid nodular lesion.

The fact that the histology of the halo sign was composed not only of closely packed macrophages in the alveoli with thickened alveolar septa but also of intra-alveolar granulomas, and that some granulomas had polypoid structures that arose from the wall of the peripheral airway, is worthy of special mention. Intra-alveolar epithelioid granulomas were previously reported in sarcoidosis by SHIGEMATSU et al. [2] and YANAGAWA et al. [5], but their histological descriptions were dependent on TBLB specimens. In our report, we found intraalveolar sarcoid granulomas in the surgically biopsied specimen. In addition, we also demonstrated that the surface of the granulomas was partly covered with epithelial cells, which raises the possibility that intra-alveolar granulomas grow in the interstitial tissue and then protrude into peripheral airways.

The differential diagnosis includes tumour-related sarcoid reactions and other granulomatous lung diseases, including Wegener's granulomatosis. The patient was a housewife who was not exposed to any occupational or environmental dust. The granulomas described in this report were well formed, non-necrotising and had Langhans' giant cells in the lung parenchyma, not in the regional lymph nodes. No geographical necrosis, which is characteristic of Wegener's granulomatosis, was found in the biopsy specimen. In addition, there was neither renal nor upper airway involvement and both serum myeloperoxidase- and proteinase 3-antineutrophil cytoplasmic antibody were negative.

In conclusion, we have shown for the first time that the CT halo sign in sarcoidosis histologically represents intra-alveolar sarcoid granulomas and aggregates of macrophages in the alveolar spaces with thickened alveolar septa. Further studies are needed to elucidate the genesis and development of pulmonary sarcoidosis.

\section{T. Harada*\#, K. Nabeshima", T. Matsumoto*, T. Akagi*, M. Fujita* and K. Watanabe*}

*Dept of Respiratory Medicine, and \#Dept of Pathology, Fukuoka University School of Medicine, Fukuoka, Japan.

Correspondence: K. Watanabe, Dept of Respiratory Medicine, Fukuoka University School of Medicine, Fukuoka, 814-0180, Japan. E-mail: watanabe@fukuoka-u.ac.jp

Statement of Interest: None declared.

\section{REFERENCES}

1 Sahn SA, Schwarz MI, Lakshminarayan S. Sarcoidosis: the significance of an acinar pattern on chest roentgenogram. Chest 1974; 65: 684-687.

2 Shigematsu N, Emori K, Matsuba K, et al. Clinicopathologic characteristics of pulmonary acinar sarcoidosis. Chest 1978; 73: 186-188.

3 Marten K, Rummeny EJ, Engelke C. The CT halo: a new sign in active pulmonary sarcoidosis. Br J Radiol 2004; 77: 1042-1045.

4 Poletti V, Patelli M, Spiga L, et al. Transbronchial lung biopsy in pulmonary sarcoidosis. Is it an evaluable method in detection of disease activity? Chest 1986; 89: 361-365.

5 Yanagawa T, Okada J, Mochida A, et al. A case of sarcoidosis acutely aggravated with high fever and diffuse interstitial pulmonary infiltrates. Nippon Kokyuki-Gakkai Zasshi 2001; 39: 377-382.

DOI: $10.1183 / 09031936.00029509$ 